\title{
Laser-induced tissue remodeling within the tympanic membrane
}

Sophie A. L. Schacht

Patricia Stahn

Marius Hinsberger

Bernhard Schick

Gentiana I. Wenzel 


\title{
Laser-induced tissue remodeling within the tympanic membrane
}

\author{
Sophie A. L. Schacht, ${ }^{\star}$ Patricia Stahn, Marius Hinsberger, Bernhard Schick, and Gentiana I. Wenzel \\ University of Saarland, Department of Otorhinolaryngology, Homburg, Germany
}

\begin{abstract}
The tympanic membrane (TM) separates the outer ear from the tympanic cavity. Repeated pathologies can permanently decrease its tension, inducing conductive hearing loss and adhesive processes up to cholesteatoma. The current main therapy is its surgical reconstruction. Even though lasers have been proposed to tighten atrophic TMs, details of this effect, specifically histological analyses, are missing. We therefore used laser pulses to induce TM collagen remodeling in an animal model to compare the histological and electrophysiological effects of different applied laser intensities before entering clinical studies. We irradiated Fuchsin-stained areas of the TM in anesthetized mice with 532-nm laser-pulses of $10 \mathrm{~mW}$ for $30 \mathrm{~s}(0.3 \mathrm{~J}), 25 \mathrm{~mW}$ for $30 \mathrm{~s}(0.75 \mathrm{~J})$ or $50 \mathrm{~mW}$ for $30 \mathrm{~s}(1.5 \mathrm{~J})$ monitoring hearing with auditory brainstem responses (ABRs). The mice were sacrificed after 2 to 8 weeks and histologically analyzed. An increase in the TM thickness within the defined, stained, and irradiated areas could be observed after 4 weeks. Polarized light microscopy and transmission electron microscopy demonstrated the tissue volume increase majorly due to new collagen-fibrils. Directly after irradiation, ABR thresholds did not increase. We herein demonstrate a controlled laser-induced collagen remodeling within defined areas of the TM. This method might be the prophylactic solution for chronic inflammatory ear pathologies related to decreased TM tension. (- The Authors. Published by SPIE under a Creative Commons Attribution 3.0 Unported License. Distribution or reproduction of this work in whole or in part requires full attribution of the original publication, including its DOI. [DOI: 10.1117/1.JBO.23.12.121614]
\end{abstract}

Keywords: tympanic membrane; remodeling; collagen; hearing; laser irradiation; mice.

Paper 180388SSR received Jun. 27, 2018; accepted for publication Oct. 19, 2018; published online Nov. $29,2018$.

\section{Introduction}

The tympanic membrane (TM) separates the outer ear canal from the middle ear. ${ }^{1}$ Its function is to collect the sound that has been transmitted through the ear canal, amplify it and transmit it further through the ossicular chain to the inner ear. ${ }^{2,3}$

The TM layer facing the outer ear canal is represented by the slightly keratinized epithelium (epidermal layer) and the subepidermal layer of loose connective tissue. The median layer, called lamina propria, is the collagen rich structure of the TM consisting of a radiar (external) and circular (internal) collagen layer. The predominant type of collagen within the pars tensa is collagen type II. ${ }^{4}$ The TM layer facing the tympanic cavity consists of the monolayered epithelium (mucosal layer), which covers the tympanic cavity as well [Fig. 1(c)].$^{5-8}$

As a common deficiency after chronic affections involving the eardrum and TM perforations, the TM often heals with atrophic scars. These atrophic parts of the TM are thinner than the nonaffected TM and have a deficiency in fibroblasts and the collagen layer. ${ }^{9,10}$

Depending on the dimensions of the atrophic area of the TM and the ventilation of the tympanic cavity, different conditions appear: (1) localized atrophic scar, (2) TM retraction pocket, and (3) atelectasis of the tympanic cavity. ${ }^{11-15}$

To prevent the development of cholesteatomas, the further major and life endangering complication of these pathologies, and correct conductive hearing loss, the surgical intervention (tympanoplasty) is currently the state-of-the-art therapy complemented with measures that improve the Eustachian tube function.

\footnotetext{
*Address all correspondence to: Sophie A. L. Schacht, E-mail: sophie
} schacht@gmx.de; Gentiana I. Wenzel, E-mail: Gentiana.Wenzel@uks.eu
Laser irradiation of different types of tissues-such as the basilar membrane of the cochlea, ${ }^{16}$ the cornea, ${ }^{17}$ the femoropatellar joint capsule, ${ }^{18,19}$ the medial collateral ligament, ${ }^{20}$ the periodontal ligament, ${ }^{21}$ and the skin ${ }^{22}$-is known to induce conformational changes in the structural collagen. Although in the first period after irradiation, a decrease in the tissue stiffness can be observed, ${ }^{18,20}$ a consequent increase in the tissue stiffness follows after days to years due to collagen remodeling. ${ }^{20,23-25}$ The question arises therefore if this method could be used for collagen remodeling within the TM as well.

Kurkowa and Goode ${ }^{13}$ first proposed the use of lasers to tighten atrophic $\mathrm{TM}^{13}$ demonstrating the contraction of these pockets in explanted human temporal bones. Later, Ostrowski and Bojrab, ${ }^{14}$ Brawner et al., ${ }^{15}$ and Ryen and Kaylie ${ }^{12}$ performed the first clinical studies. However, this idea did not make it to the routine therapeutic strategy due to the need of further studies. ${ }^{12}$ We therefore proposed to assess the laser treatment to induce collagen remodeling within the TM in an animal model and use the advantage of comparing the effects of different applied laser intensities from histological and electrophysiological point of view before entering clinical studies. Additionally, we applied pigments to reduce the light energy needed and exactly define the borders of the TM areas to be remodeled, while leaving the rest unaffected. This could be the prophylactic intervention in an early stage of the disease, that would stop the process and avoid a later surgical procedure, having the advantage of very confined tissue remodeling to the affected area, using lasers.

\section{Material and Methods}

Wenzel et al. ${ }^{26}$ demonstrated that 2 weeks after laser irradiation of the basilar membrane within the cochlea, collagen remodeling, 


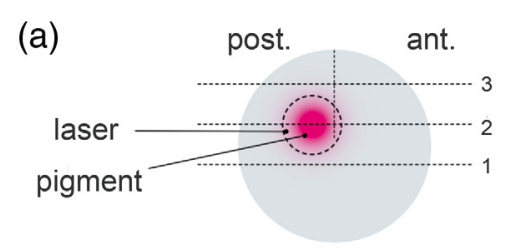

(b)
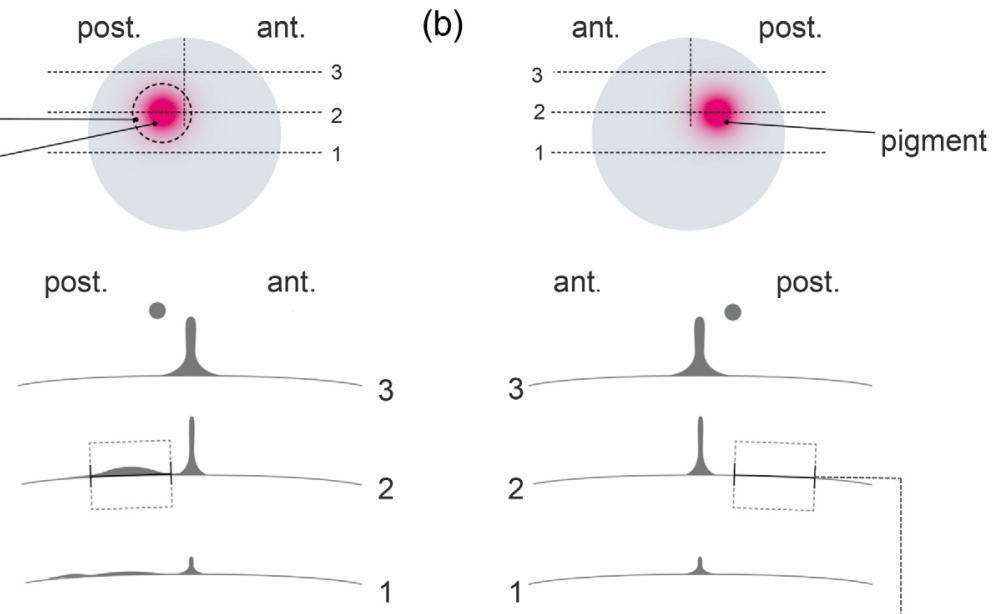

(c)

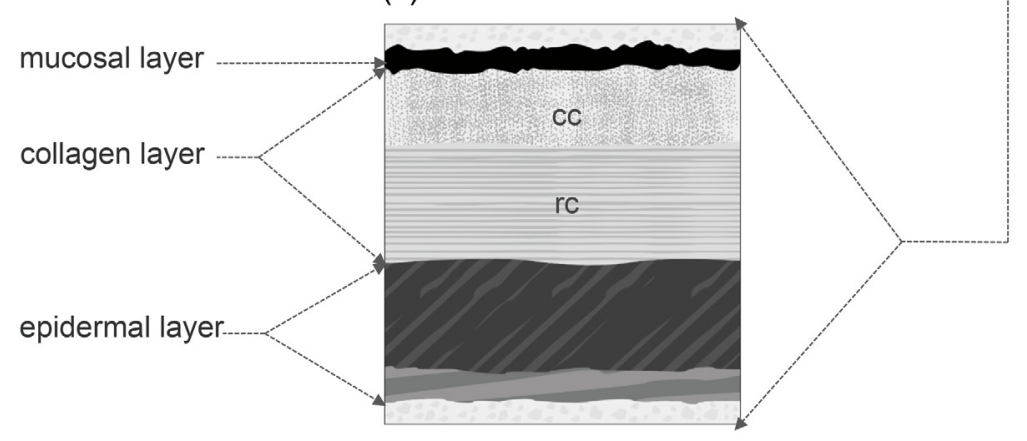

Fig. 1 Schematic illustration of the TM and the sections (1-3) used from each ear included in our study. (a) Left irradiated TM. The dashed line box marks the stained and irradiated area of the TM. In both images, the perpendicular dark gray line represents the first ossicle. The squared dashed box represents the area that has been stained (fuchsia color) and irradiated. The analyzed sections are numbered 1-3. (b) Right stained but not irradiated TM of the control ear. (c) Simplified structure of the TM as represented in the TEM, divided in the inner mucosal layer, the collagen layer with circular-(cc) and radiar-(rc) organized collagen and the outer epidermal layer.

and new collagen deposition could be observed. Based on these studies, we designed our herein presented method for laser irradiation of the TM and its electrophysiological and histological results in an animal model.

\subsection{Animals}

85 female CD1 albino mice, weighing 20 to $30 \mathrm{~g}$ at the age of 5 to 8 weeks were used for this study. Out of these, the temporal bones of 74 animals were processed for the analyses with transmitted light and polarized light microscopy, and 11 animals for transmission electron microscopy (TEM). Auditory brainstem responses (ABRs) were recorded to monitor hearing.

\subsection{Surgical Technique}

The "Saarländische Landesamt für Verbraucherschutz" approved the study protocol in accordance with the "Deutschen Tierschutzgesetz und der Richtlinie des Rates der europäischen Gemeinschaft zum Schutz der für experimentelle Zwecke verwendeten Tiere."

The mice were anesthetized with an intraperitoneal injection $(0.1 \mathrm{ml} / \mathrm{g} \mathrm{BW})$ of $2.125 \mathrm{ml} 0.9 \%$ isotone solution of sodium chloride, $0.25 \mathrm{ml}$ ketamine, and $0.125 \mathrm{ml}$ rompun. During the experiment, the body temperature was maintained at $38^{\circ} \mathrm{C}$ using an electric heating pad.

After trimming the hair around the ear, we performed a 2-mm long incision beginning at the incisura intertragica and extending it along the cartilaginous outer ear canal exposing the TM on both sides. After recording ABR, the external ear was anchored with sutures to expose the entire TM.

To enhance the laser energy absorption and achieve optical selectivity for the proposed defined part of the TM, we stained the target area of the posterior (post) superior quadrant (diameter ca. $400 \mu \mathrm{m})$ with the pigment Fuchsin $(0.5 \%$ solution with alcohol, pharmacy, Saarland University), a water-soluble solution that has its maximal optical absorption at 455 to $600 \mathrm{~nm}^{27,28}$ We stained the contralateral ear as well as a control. The anterior (ant.) part of the TM was, on both sides, not touched. After staining, we irradiated the marked area on the left TM (Fig. 1).

The ABR thresholds were then recorded again and the incisions on both ears sutured. Until fully awake, the mouse was monitored and put under an infrared lamp to protect it from hypothermia.

After 14, 28, or 56 days, according to the respective test group, the mice were anesthetized again, the incisions on both ears reopened and final ABR thresholds were measured. The animals were then sacrificed through cervical dislocation 
Table 1 Summary of the analyzed animals with focus onto the irradiation duration, intensity, and total dose of laser energy applied as well as the day of sacrifice.

Day of sacrifice and number of animals

Intensity, duration of irradiation, and total dose of laser energy

\begin{tabular}{llll}
\hline $10 \mathrm{~mW}$ for $30 \mathrm{~s} \sim 0.3 \mathrm{~J}$ & $n=6$ & $n=9$ & $n=6$ \\
$25 \mathrm{~mW}$ for $30 \mathrm{~s} \sim 0.75 \mathrm{~J}$ & $n=7$ & $n=11$ & $n=8$ \\
$50 \mathrm{~mW}$ for $30 \mathrm{~s} \sim 1.5 \mathrm{~J}$ & $n=8$ & $n=12$ & $n=8$ \\
\hline
\end{tabular}

under deep anesthesia. The petrous part of the temporal bone was extracted $e x$ vivo and the tympanic cavity opened and perfused with fixative.

\subsection{Laser Irradiation}

We used a 532-nm pulsed neodynium-doped yttrium orthovanadate $\left(\mathrm{Nd}: \mathrm{YVO}_{4}\right.$ ) (Xiton Photonics $\mathrm{GmbH}$, Kaiserslautern) as the laser source. The pulsed laser light was applied through a laser fiber with a diameter of $365 \mu \mathrm{m}$ that we directed to the stained area of the TM with a micromanipulator. We estimated the distance as close as visible possible to the stained area, without touching the TM, giving an irradiation diameter of $\sim 400 \mu \mathrm{m}$ on the TM. The final position adjustment has been performed using a continuous waved pilot laser, with a power of $0.1 \mathrm{~mW}$, for a maximum of $30 \mathrm{~s}$ (Fig. 1). We then irradiated the stained area of the TM for $30 \mathrm{~s}$ with a power of either $10 \mathrm{~mW}(0.3 \mathrm{~J})$, $25 \mathrm{~mW}(0.75 \mathrm{~J})$, or $50 \mathrm{~mW}(1.5 \mathrm{~J})$ at a laser repetition rate of $2 \mathrm{kHz}$ (Table 1).

\subsection{Electrophysiological Monitoring: $A B R$}

We used a digital signal processing system that generated sine wave stimuli (Agilent 33500 B Series Trueform Waveform Generator, Keysight Technologies $\mathrm{GmbH}$, Germany) and delivered them through a free-field speaker (Beyerdynamic $\mathrm{GmbH} \&$ Co. KG) placed in front of the mouse at $5 \mathrm{~cm}$ distance to each ear. With this system, we recorded click- and frequency-specific ABRs using four needle electrodes: one on each mastoid, one at the vertex (reference), and one at the base of the tail (ground). The recorded signals were then amplified through the biosignal amplifier (g.USBamp, g.tec medical engineering $\mathrm{GmbH}$, Austria), digitized at $19.2 \mathrm{kHz}$ and digitally filtered to obtain the frequencies in the 300 to $2500 \mathrm{~Hz}$ range. The stimulus intensities ranged from 0 to $80 \mathrm{~dB}$ SPL increased in $10 \mathrm{~dB}$ steps at 2 , 4, 8, 12, 16, 20, 32, 48, and $80 \mathrm{kHz}$.

We estimated the hearing thresholds for each recording visually, offline and defined it as the lowest intensity, where the Jewett complex was identifiable. Additionally, within the click ABR data, we measured the amplitude and latency of the wave I peak to peak. ${ }^{29}$

\subsection{Transmitted Light Microscopy}

We explanted the temporal bones, opened the tympanic cavity, and fixated the samples with a $4 \%$ formaldehyde for 2 days. We performed the decalcification in $20 \%$ EDTA with $100 \%$ citric acid ( $\mathrm{pH}$ 7.2) for 4 days, dehydrated them in ascending ethanol series of $70 \%$ and $100 \%$ (360 min), placed them in $100 \%$ Xylol (180 min), and finally embedded them in paraffin. Afterward, we cut the blocks in $8 \mu \mathrm{m}$ sections and stained with hematoxylin-eosin to get a first impression of the anatomy. We then used the picrosirius red staining (Sigma-Aldrich Chemie GmbH, München) (MORPHISTO ${ }^{\circledR}$ Evolutionsforschung und Anwendung $\mathrm{GmbH}$, Frankfurt) to analyze the morphological changes within the TM.

For transmission microscopy, we used three to four slides of each ear. The interval between each utilized slide-series was $\sim 210 \mu \mathrm{m}$. We then measured the thickness of the posterior part of the TM from the left (irradiated) and the right (nonirradiated) ear as a control (Fig. 1 dashed squares).

\subsection{Polarized Light Microscopy}

We used picrosirius red and added hemotoxylin to enhance the natural birefringence of collagen and visualize regions with just a minimal quantity of collagen. ${ }^{30,31}$ Picrosirius red is an elongated, birefringent molecule, which binds parallel to collagen molecules being used to identify and characterize collagen structures within biological tissues. . $^{17,30,32-34}$

The sections were then visualized and imaged under polarized light microscopy. To ensure that the settings of the microscope were always the same, we started with a control ear and rotated the polarizers until the birefringence of the tissue was maximized. Furthermore, the remaining microscope settings were optimized. Once this was done, the polarizers were locked and the light exposure, the aperture size, the position were also held constant for all the other slides and images captured.

We used ImageJ (National Institutes of Health) to quantify the highest intensity of birefringence analyzing areas of 3000 to 4000 pixels within the irradiated and the corresponding control TM.

\subsection{Transmission Electron Microscopy}

For a more detailed analysis of the structural changes within the TM, 4 weeks after irradiation, we used TEM. The explanted petrous bones were fixed for 1 day in $1 \%$ glutaraldehyd in $0.1 \mathrm{M}$ cacodylate buffer containing $1 \%$ paraformaldehyde and $\mathrm{H}_{2} \mathrm{O}$ at a pH 7.2 to 7.4 and afterward decalcified in 20\% EDTA for 6 days, washed with $0.1 \mathrm{M}$ cacodylat buffer, and fixed with $0.2 \%$ osmium tetroxide. As a next step, the samples were washed in distillated $\mathrm{H}_{2} \mathrm{O}$ and gradually dehydrated from $80 \%$ to $100 \%$ ethanol and three times stained with acetone, two times with epon (3:1, 1:1, 1:3), and subsequently embedded in pure epon followed by polymerization for 2 days at $60^{\circ} \mathrm{C}$. After cutting them in about 70-nm thin sections with an ultramicrotome (LEICA EM UC7, (C) 2015 Leica Biosystems, Nussloch), they were positioned on a copper grid, contrasted with $2 \%$ uranyl acetate, postcontrasted with lead citrate, and analyzed with a transmission electron microscope (FEI TECNAI 12, The Netherlands).

\subsection{Statistical Methods}

We used SPSS and performed all tests at an alpha level of 0.05. The hearing thresholds for the click stimulation as well as for the frequency-specific stimulation, as described in Sec. 2.2, were analyzed with a nonparametric test (Friedman). Additionally, we conducted a repeated measure analysis of variance (rmANOVA) for the factor "power" and an rmANOVA for the factor "weeks" 
using Huynh-Feldt correction for the degrees of freedom. For the analysis of wave I of the ABR recordings, the TM thickness, and the TM polarization, we conducted a one factorial ANOVA, followed by a Bonferroni posthoc test to compare the individual groups. We also performed a Levene test of variance homogeneity. If this turned to be significant, we performed a more robust test, the Welch ANOVA, followed by a Games-Howell procedure as posthoc test. Additionally, in the cases when the data were not distributed normally, we performed a nonparametric test, the Friedman test, followed by a Bonferroni posthoc test.

\section{Results}

\subsection{Transmission Light Microscopy}

The thickness of the TM increased with increasing intensity of irradiation (Fig. 2). This demonstrated to be valid and statistically significant for the animals exposed to $25 \mathrm{~mW}$ for $30 \mathrm{~s}(0.75 \mathrm{~J})$ and $50 \mathrm{~mW}$ for $30 \mathrm{~s}(1.5 \mathrm{~J})$ independent of the time postexposure. The animals exposed to $10 \mathrm{~mW}$ for $30 \mathrm{~s}$ $(0.3 \mathrm{~J})$ did not have any increase in TM thickness in comparison to the contralateral nonexposed ear. To quantify these changes, we subtracted from the thickness of the stained and irradiated area of the (left) TM, the thickness of the same area of the stained nonirradiated (right) ear in each animal, and plotted the averaged results of these differences (Fig. 3). The maximal increase in TM thickness could be observed for the group of mice irradiated with $50 \mathrm{~mW}$ for $30 \mathrm{~s}(1.5 \mathrm{~J})$ and sacrificed after 2 weeks. Even though the trend was toward an increase in thickness with the increase in optical power applied, this change was, however, statistically significant just between $10 \mathrm{~mW}$ for $30 \mathrm{~s}(0.3 \mathrm{~J})$ and $25 \mathrm{~mW}$ for $30 \mathrm{~s}(0.75 \mathrm{~J})$ or
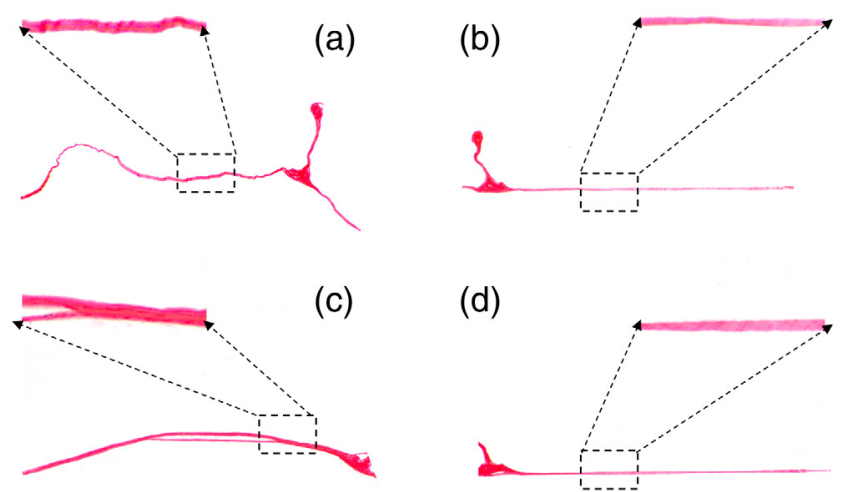

(d)
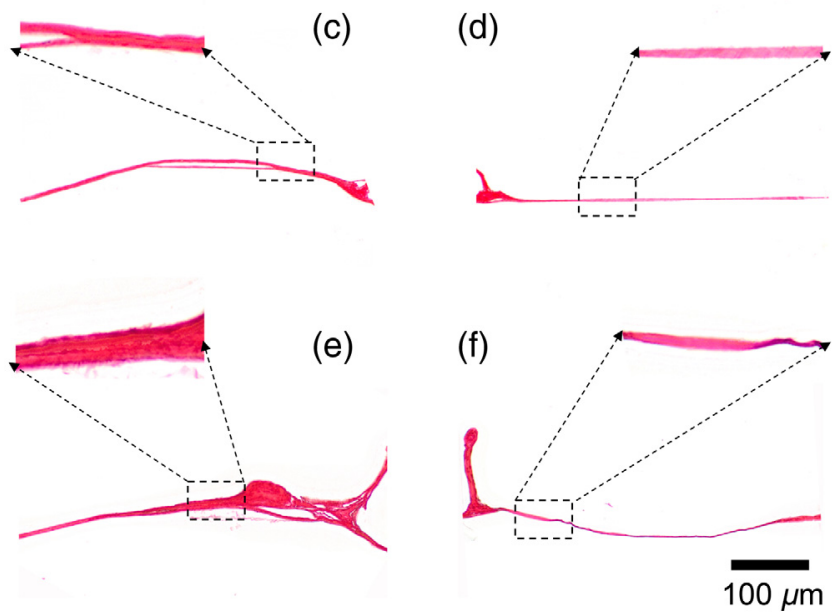

Fig. 2 Transmission light microscopy. Four weeks after irradiation with (a) $5 \mu \mathrm{J} /$ pulse $(10 \mathrm{~mW}$ for $30 \mathrm{~s} \sim 0.3 \mathrm{~J}$ ); (c) $12.5 \mu \mathrm{J} /$ pulse (25 mW for $30 \mathrm{~s} \sim 0.75 \mathrm{~J})$; (e) $25 \mu \mathrm{J} /$ pulse $(50 \mathrm{~mW}$ for $30 \mathrm{~s} \sim 1.5 \mathrm{~J}) ;(\mathrm{a}),(\mathrm{c}),(\mathrm{e})$ (upper posterior quadrant of the irradiated left TM) in comparison with the contralateral ear; (b), (d), (f) (upper posterior quadrant of the nonirradiated right TM).
$10 \mathrm{~mW}$ for $30 \mathrm{~s}(0.3 \mathrm{~J})$ and $50 \mathrm{~mW}$ for $30 \mathrm{~s}(1.5 \mathrm{~J})$ but not significant between $25 \mathrm{~mW}$ for $30 \mathrm{~s}(0.75 \mathrm{~J})$ and $50 \mathrm{~mW}$ for $30 \mathrm{~s}$ $(1.5 \mathrm{~J})$. This effect could be observed at all time points of sacrifice 2 , 4, or 8 weeks.

The mice who were sacrificed after 2 weeks demonstrated a significant increase in the TM-thickness between the groups irradiated with $10 \mathrm{~mW}$ for $30 \mathrm{~s}(0.3 \mathrm{~J})$ compared to the once irradiated with $25 \mathrm{~mW}$ for $30 \mathrm{~s}(0.75 \mathrm{~J})(p=0.007)$ and $50 \mathrm{~mW}$ for $30 \mathrm{~s}(1.5 \mathrm{~J})(p=0.000)$, but no significant change between the mice irradiated with a power of $25 \mathrm{~mW}$ for $30 \mathrm{~s}$ $(0.75 \mathrm{~J})$ to the group irradiated with a power of $50 \mathrm{~mW}$ for $30 \mathrm{~s}(1.5 \mathrm{~J})(p=0.090)$.

The mice sacrificed after 4 weeks demonstrated a significant increase of the TM thickness between the group irradiated with $10 \mathrm{~mW}$ for $30 \mathrm{~s}(0.3 \mathrm{~J})$ to the groups irradiated with $25 \mathrm{~mW}$ for $30 \mathrm{~s}(0.75 \mathrm{~J})(p=0.001)$ and $50 \mathrm{~mW}$ for $30 \mathrm{~s}(1.5 \mathrm{~J})$ $(p=0.002)$. Again, no significant TM thickness increase could be observed between the mice exposed to $25 \mathrm{~mW}$ for $30 \mathrm{~s}(0.75 \mathrm{~J})$ and the ones exposed to $50 \mathrm{~mW}$ for $30 \mathrm{~s}(1.5 \mathrm{~J})$ $(p=0.165)$.

Similar to the mice sacrificed after 4 weeks, the mice sacrificed after 8 weeks demonstrated a significant change in TM thickness between the $10 \mathrm{~mW}$ for $30 \mathrm{~s}(0.3 \mathrm{~J})$ irradiated mice to the $25 \mathrm{~mW}$ for $30 \mathrm{~s}(0.75 \mathrm{~J})(p=0.000)$, and $50 \mathrm{~mW}$ for $30 \mathrm{~s}(1.5 \mathrm{~J})(p=0.002)$ animals, however, no significant change in the TM thickness of mice exposed to $25 \mathrm{~mW}$ for $30 \mathrm{~s}(0.75 \mathrm{~J})$ compared the ones exposed to $50 \mathrm{~mW}$ for $30 \mathrm{~s}(1.5 \mathrm{~J})$ $(p=0.305)$ (Fig. 3).

Additionally, we compared the sections cranial (1) and caudal (3) to the stained and irradiated area (2) of the TM (Fig. 1). The analysis demonstrated that a tissue proliferation was induced just in the area of the stained and irradiated part of the TM $(p<0.005)$. The parts above and underneath this area were not significant different to their stained and nonirradiated controls (contralateral ear) $(p=1.000)$.

\subsection{Polarized Light Microscopy}

We analyzed the TMs birefringence as an estimation of the build/remodeled collagen using polarized microscopy, as described in Sec. 2.6 (Fig. 4), and compared the birefringence of the stained and irradiated area of the left TM with the same area of the control-right TM (stained and not irradiated).

The mice irradiated with $25 \mathrm{~mW}$ for $30 \mathrm{~s}(0.75 \mathrm{~J})$ or $50 \mathrm{~mW}$ for $30 \mathrm{~s}(1.5 \mathrm{~J})$ demonstrated after 4 and 8 weeks an increase of the TM birefringence. The statistical analysis demonstrated that this increase was statistical significant 4 weeks postirradiation: $(p=0.045)$ for $25 \mathrm{~mW}$ for $30 \mathrm{~s}(0.75 \mathrm{~J})$ and $(p=0.019)$ for $50 \mathrm{~mW}$ for $30 \mathrm{~s}(1.5 \mathrm{~J})$, however, not significant between the animals irradiated with $25 \mathrm{~mW}$ for $30 \mathrm{~s}(0.75 \mathrm{~J})$ to the $50 \mathrm{~mW}$ for $30 \mathrm{~s}(1.5 \mathrm{~J})(p=1.000)$.

The incubation time of 2 weeks did not induce a statistical significant change in the TM-polarization either $(p=0.568)$ (Fig. 5).

Within the mice groups, which were sacrificed after 8 weeks, a significant increase in birefringence could be demonstrated between the mice group irradiated with $10 \mathrm{~mW}$ for $30 \mathrm{~s}$ $(0.3 \mathrm{~J})$ and the ones irradiated with $25 \mathrm{~mW}$ for $30 \mathrm{~s}(0.75 \mathrm{~J})$ $(p=0.024)$ as well, however, no significant change between the $25 \mathrm{~mW}$ for $30 \mathrm{~s}(0.75 \mathrm{~J})$ irradiation and the ones irradiated with $50 \mathrm{~mW}$ for $30 \mathrm{~s}(1.5 \mathrm{~J})(p=1.000)$. Interestingly, despite the clear trend toward increase in birefringence with increasing energy, the statistical analysis revealed no significant increase in 


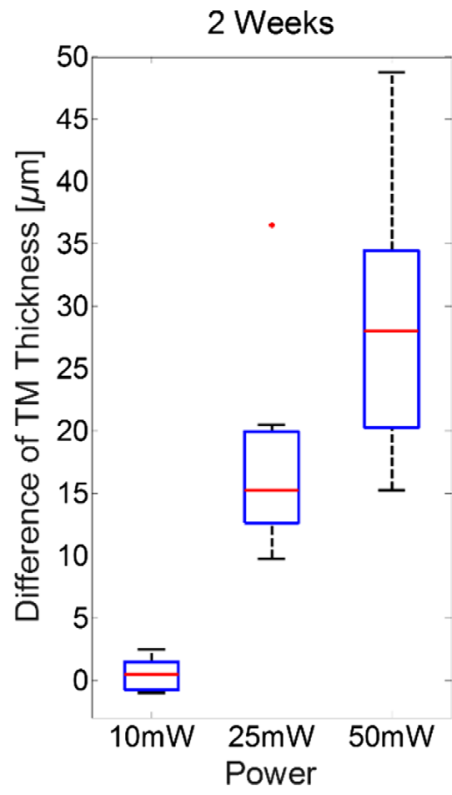

(a)

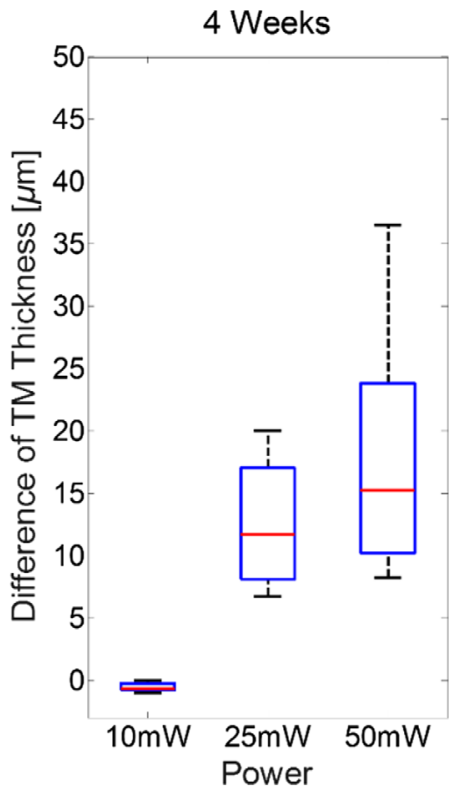

(b)

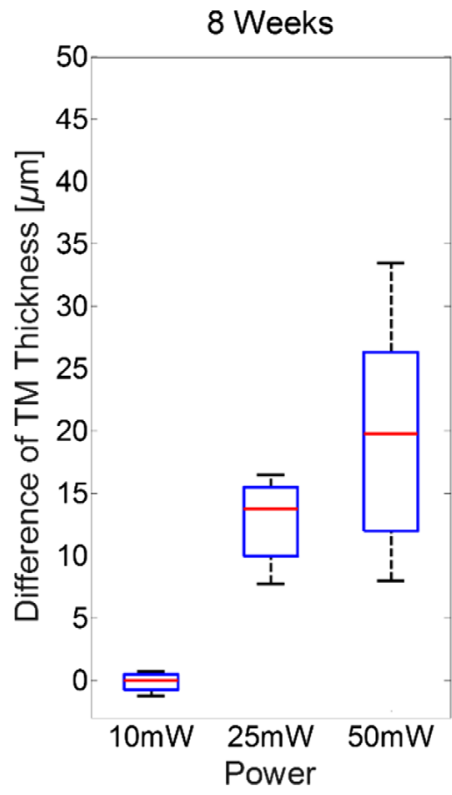

(c)

Fig. 3 Comparison between the analyzed mice groups presenting their TM thickness of section (2) (for reference, see Fig. 1) plotted on the $y$ axis relative to the irradiation levels of $10 \mathrm{~mW}$ for $30 \mathrm{~s} \sim 0.3 \mathrm{~J}$, $25 \mathrm{~mW}$ for $30 \mathrm{~s} \sim 0.75 \mathrm{~J}$ or $50 \mathrm{~mW}$ for $30 \mathrm{~s} \sim 1.5 \mathrm{~J}$ ( $x$ axis) for the different incubation times of (a) 2, (b) 4, and (c) 8 weeks.

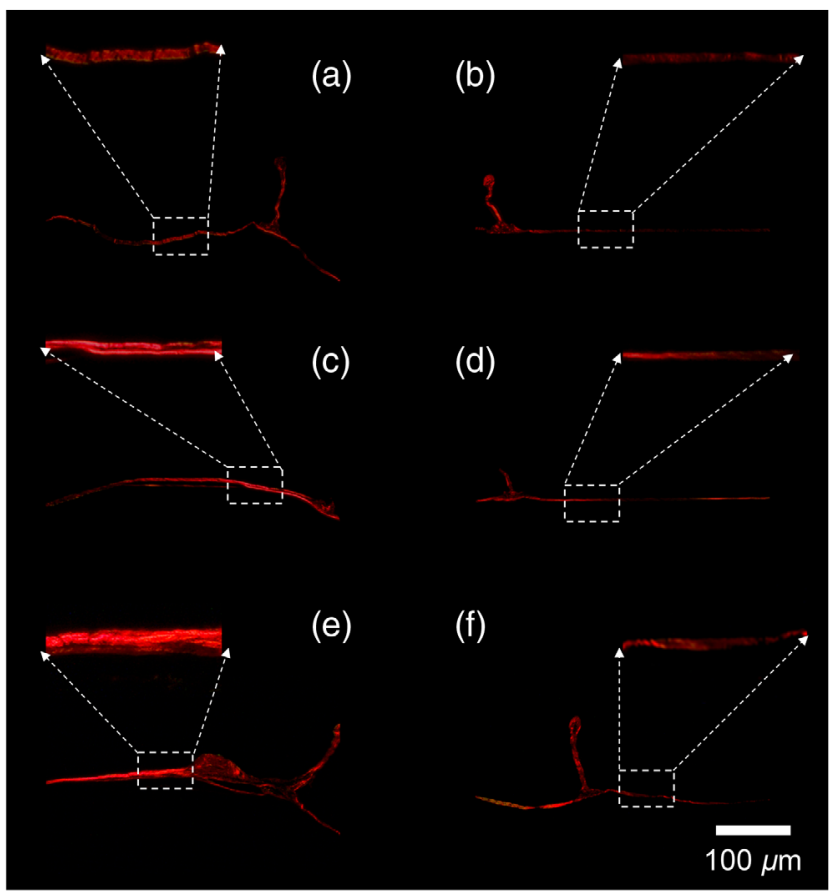

Fig. 4 Polarized light microscopy. Four weeks after irradiation of the upper posterior quadrant of the stained and irradiated left TM with (a) $5 \mu \mathrm{J} /$ pulse $(10 \mathrm{~mW}$ for $30 \mathrm{~s} \sim 0.3 \mathrm{~J})$; (c) $12.5 \mu \mathrm{J} /$ pulse $(25 \mathrm{~mW}$ for $30 \mathrm{~s} \sim 0.75 \mathrm{~J}$ ); (e) $25 \mu \mathrm{J} /$ pulse $(50 \mathrm{~mW}$ for $30 \mathrm{~s} \sim 1.5 \mathrm{~J})$ (b), (d), (f) in comparison with the upper posterior quadrant of the stained nonirradiated right TM (contralateral ear).

birefringence between the mice irradiated with $10 \mathrm{~mW}$ for $30 \mathrm{~s}$ $(0.3 \mathrm{~J})$ and $50 \mathrm{~mW}$ for $30 \mathrm{~s}(1.5 \mathrm{~J})(p=0.135)$. After exposing the stained TMs to $10 \mathrm{~mW}$ for $30 \mathrm{~s}(0.3 \mathrm{~J})$, the increase of birefringence was not statistically significant either (Fig. 5).
Over time the increase in birefringence was significant within the first 4 weeks for the animals irradiated with $25 \mathrm{~mW}$ for $30 \mathrm{~s}$ $(0.75 \mathrm{~J})(p=0.001)$ and $50 \mathrm{~mW}$ for $30 \mathrm{~s}(1.5 \mathrm{~J})(p=0.000)$ followed by a stagnation within the next 4 weeks (nonsignificant increase in birefringence in the mice irradiated with a power of $25 \mathrm{~mW}$ for $30 \mathrm{~s}(0.75 \mathrm{~J})(p=0.612)$ and $50 \mathrm{~mW}$ for $30 \mathrm{~s}$ $(1.5 \mathrm{~J})(p=1.000))$. The animals irradiated with $10 \mathrm{~mW}$ for $30 \mathrm{~s}(0.3 \mathrm{~J})$ had no significant increase in birefringence $(p=0.773)$.

As expected, a significant increase in birefringence was observed in the main stained and irradiated Sec. 2 of the TM ( $p=0.003$ when comparing the irradiated to the nonirradiated control and $p=0.029$ for the difference between Secs. 1 and 2 and $p=0.002$ for the comparison of Secs. 2 and 3). The birefringence within the area of the outer Secs. 1 and 3 did not differ significantly $(p=0.831)$.

\subsection{Transmission Electron Microscopy}

The nonirradiated TMs [Figs. 6(b), 6(d), and 6(f)] revealed the typical three layered structure. ${ }^{7,8}$ Within the TM irradiated with $10 \mathrm{~mW}$ for $30 \mathrm{~s}(0.3 \mathrm{~J})$ [Fig. 6(a)], we observed a slight change in the radial organized collagen layer, which seemed to be arranged more loosely and was slightly interspersed with circular collagen fibers. Within the TM irradiated with $25 \mathrm{~mW}$ for $30 \mathrm{~s}(0.75 \mathrm{~J})$ [Fig. 6(c)], the new circular structured collagen fibers appeared to be at least doubled compared to the contralateral, nonirradiated TMs. In addition, the ratio between the different collagen layers appeared modified with a larger increase in the circular structured collagen. Within the TM irradiated with $50 \mathrm{~mW}$ for $30 \mathrm{~s}(1.5 \mathrm{~J})$ [Fig. 6(e)], the reorganized collagen layers demonstrated a further increase in thickness and the delimitation between the different organized collagen layers was loose. 


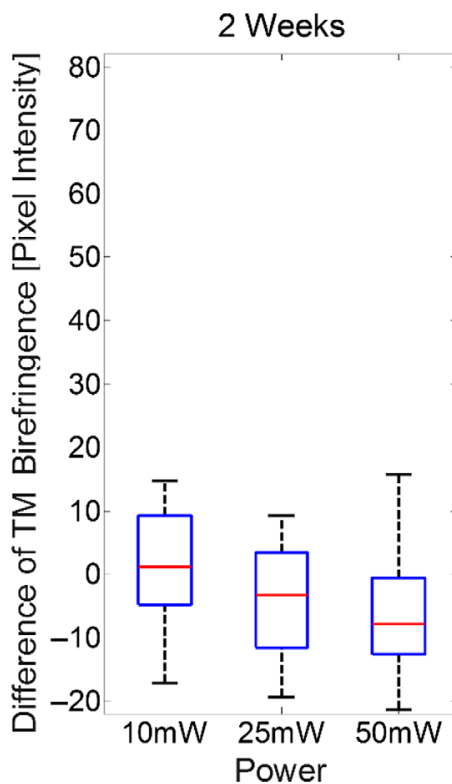

(a)

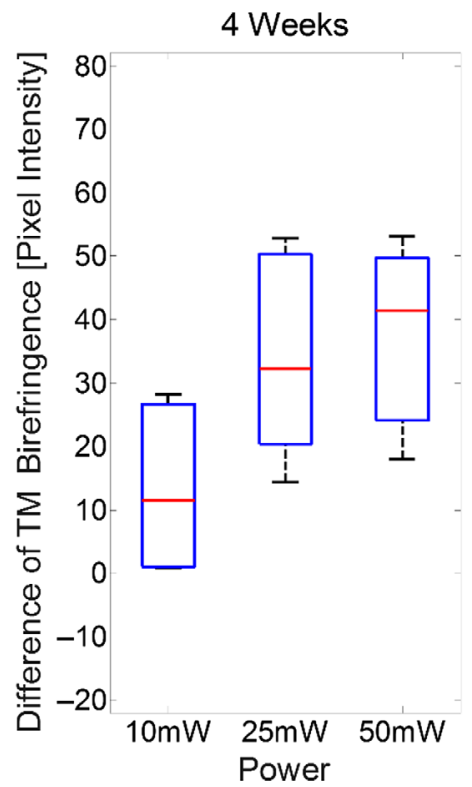

(b)

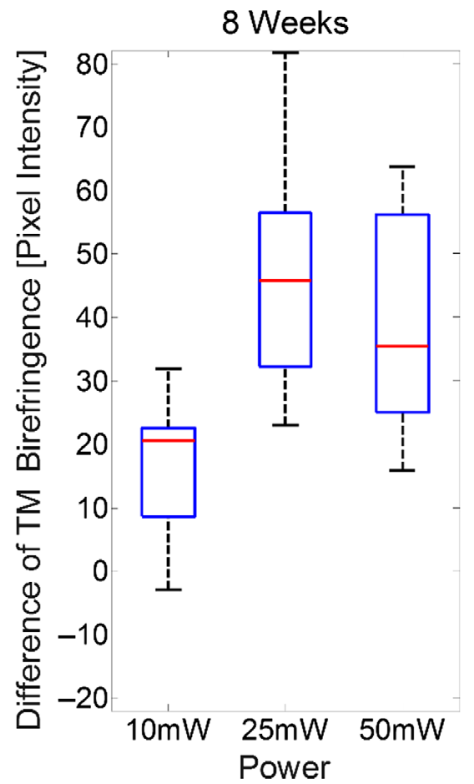

(c)

Fig. 5 Comparison between the analyzed mice groups presenting their TM birefringence (difference) of section (2) Legend: difference in birefringence plotted on the $y$ axis relative to the irradiation value of $10 \mathrm{~mW}$ for $30 \mathrm{~s} \sim 0.3 \mathrm{~J}, 25 \mathrm{~mW}$ for $30 \mathrm{~s} \sim 0.3 \mathrm{~J}$ or $50 \mathrm{~mW}$ for $30 \mathrm{~s} \sim 0.3 \mathrm{~J}$ ( $x$ axis) for the different incubation times of (a) 2, (b) 4, and (c) 8 weeks.

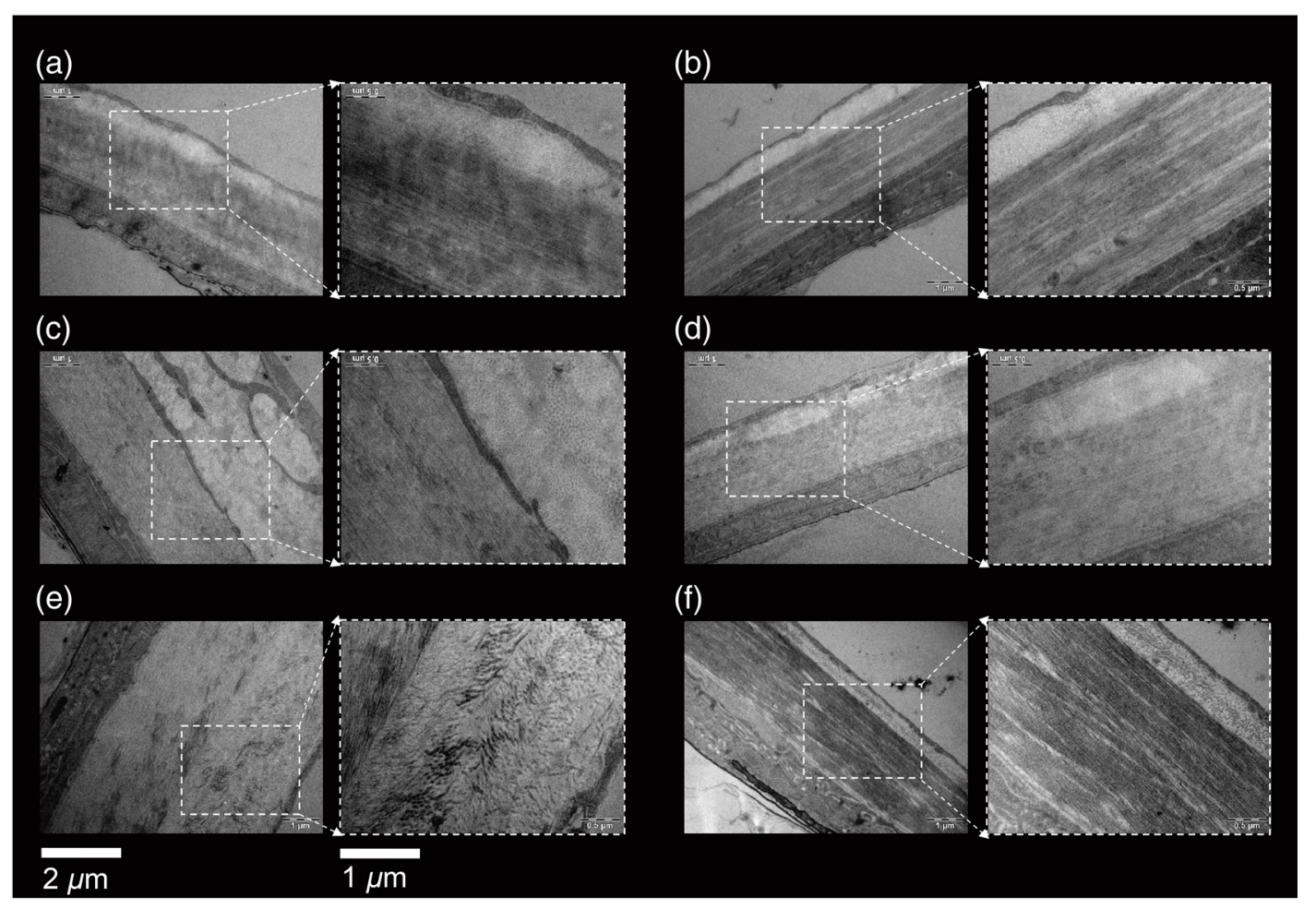

Fig. 6 Transmission electron microscopy. Four weeks after irradiation with (a) $5 \mu \mathrm{J} /$ pulse (10 $\mathrm{mW}$ for $30 \mathrm{~s} \sim 0.3 \mathrm{~J}$ ); (c) $12.5 \mu \mathrm{J} /$ pulse (25 mW for $30 \mathrm{~s} \sim 0.75 \mathrm{~J}$ ); (e) $25 \mu \mathrm{J} /$ pulse (50 mW for $30 \mathrm{~s} \sim 1.5 \mathrm{~J})$; (a), (c), (e) (upper posterior quadrant of the irradiated left TM) in comparison with the contralateral ear; (b), (d), (f) (upper posterior quadrant of the nonirradiated right TM).

\subsection{Measurement of $A B R$}

The click-ABR of each mouse demonstrated no significant threshold shift directly after irradiation. With our recording setup, no significant difference between the irradiated and the contralateral nonirradiated ear $(p=0.687)$ could be recorded. The final click-ABR recordings demonstrated a threshold shift of 10 to $40 \mathrm{~dB}$ in 56 of 75 mice that was as well observed for both ears (the irradiated and the nonirradiated ears). 
Table 2 Frequency-specific ABR: significance analysis for the individual frequencies.

\begin{tabular}{cccccccccc} 
Click & $2 \mathrm{kHz}$ & $4 \mathrm{kHz}$ & $8 \mathrm{kHz}$ & $12 \mathrm{kHz}$ & $16 \mathrm{kHz}$ & $20 \mathrm{kHz}$ & $32 \mathrm{kHz}$ & $48 \mathrm{kHz}$ & $80 \mathrm{kHz}$ \\
\hline & $p=1.000$ & $p=0.559$ & $p=0.859$ & $p=0.288$ & $p=0.826$ & $p=0.779$ & $p=0.846$ & $p=1.000$ & $p=1.000$ \\
\hline
\end{tabular}

The frequency-specific ABRs demonstrated similar results as the click-ABRs (Table 2). Twenty-five mice had in 1 to 2 frequencies a threshold shift at the second and third measurement compared to the first, both ears were affected. Nine mice were deaf from the start. The amplitude and latency of wave I did not present a major difference between the irradiated and the contralateral, not irradiated ear $(p=0.686)$.

\section{Discussion}

We herein present a preclinical study for a laser based collagen remodeling of the TM. To our knowledge, this is the first study analyzing the histological details of this process at the TM level.

We demonstrate in an animal model, that depending on the intensity of the applied laser irradiation (10 to $50 \mathrm{~mW}$ for $30 \mathrm{~s}$ $\sim 0.3$ to $1.5 \mathrm{~J}$ ) and the time of sacrifice (incubation time after irradiation), an unspecific increase in the tissue volume was induced in the TM (Figs. 2-6). The polarized light (Fig. 4) and the TEM (Fig. 6) revealed that this tissue volume change can be attributed to a laser induced collagen remodeling and collagen deposition, as described in other anatomical structures, such as the cornea, ${ }^{17}$ the femoropatellar joint capsule, ${ }^{18,19}$ the medial collateral ligament, ${ }^{20}$ the periodontal ligament, ${ }^{21,22}$ the skin, ${ }^{22}$ and the basilar membrane of the cochlea. ${ }^{26}$ To be noted that the mice irradiated with $25 \mathrm{~mW}$ for $30 \mathrm{~s}(0.75 \mathrm{~J})$ and $50 \mathrm{~mW}$ for $30 \mathrm{~s}(1.5 \mathrm{~J})$ demonstrated after 2 weeks a slight decrease of the TM-birefringence most probably through the initial disorganization of the collagen fibers in these cases. Additionally, no significant change could be observed between the animals irradiated with $25 \mathrm{~mW}$ for $30 \mathrm{~s}(0.75 \mathrm{~J})$ to the $50 \mathrm{~mW}$ for $30 \mathrm{~s}(1.5 \mathrm{~J})(p=1.000)$, demonstrating no need to increase the laser energy up to high levels in order to induce collagen remodeling.

Furthermore, we noticed that a specific amount of laserenergy is needed to induce the tissue-change we wanted. Too much energy, e.g., $50 \mathrm{~mW}$ for $30 \mathrm{~s} \mathrm{(1.5} \mathrm{J)} \mathrm{induced} \mathrm{strong} \mathrm{inflam-}$ mation observed after 2 weeks. Too low intensities (in our study, $10 \mathrm{~mW}$ for $30 \mathrm{~s} \sim 0.3 \mathrm{~J}$ ) did not have any significant effect on tissue remodeling in the herein presented study [Figs. 2(a), 3, 4(a), and 5] even though, the TEM image in Fig. 6(a) demonstrated slight disorganization of the collagen layers. Additionally, a trend toward increase in birefringence at 8 weeks for the $10 \mathrm{~mW}$ for $30 \mathrm{~s}(0.3 \mathrm{~J})$ irradiated ears could be observed that appeared however to be statistically not significant (Fig. 5).

The TEM confirmed that 4 weeks after irradiation of the TM with 10 to $50 \mathrm{~mW}$ for $30 \mathrm{~s}(0.3$ to $1.5 \mathrm{~J})$, the histological structure of the collagen layer of the TM was reorganized and an unspecific collagen increase could be observed. Within future studies, more intensities, especially between 10 and $25 \mathrm{~mW}$ for $30 \mathrm{~s}(0.3$ to $1.5 \mathrm{~J})$, that would allow a finer quantification of the needed laser parameters and of the optimal pigment in order to achieve the desired collagen deposition, while minimizing the risk of a local inflammation, are needed.

The other variable that we analyzed was the time after irradiation: 2, 4, and 8 weeks. As in previous research described, collagen remodeling is a process that takes from weeks up to several months. ${ }^{23-25}$ Shortly after the laser irradiation and especially using high intensity, an inflammation occurred. ${ }^{35}$ In our study, the collagen remodeling was significant as well starting with 4 weeks followed by stagnation within the following 4 weeks (Fig. 5).

We also noticed that the pigment (Fuchsin used in this set of experiments) was essential to improve the laser absorption within the transparent structure of the TM in our animal model as well as inducing the irradiation effects in a defined area, where the surgeon would apply the pigment not changing the original structure of the rest. Additionally, this technique enabled us to keep the amount of applied light energy at low levels compared to previous studies from Kurkowa and Goode, ${ }^{13}$ who first proposed to use lasers to tighten atrophic $\mathrm{TM}$ in human temporal bones (laser energy settings ranging from 6.5 to $19.5 \mathrm{~J} / \mathrm{cm}^{2}$ ( 2 to $6 \mathrm{~W}$ ). Ostrowski and Bojrab ${ }^{14}$ used a $\mathrm{CO}_{2}$ laser with laser power settings ranged from 0.1 to $1.0 \mathrm{~W}$ to induce contraction of TM retraction pockets in patients. Brawner et al. ${ }^{15}$ used a pulse diode potassium-titanylphosphate (KTP) laser $(532 \mathrm{~nm})$ with a diffuse beam and low power (800 to $1200 \mathrm{~mW}$ ) mentioning the use of pigments in some of their patients, and Ryen and Kaylie ${ }^{12}$ used a $\mathrm{CO}_{2}$ laser at a power of $2 \mathrm{~W}$ to treat TM atelectasis.

Even though at the beginning of the study, this was not the case, the pigment Fuchsin has been meanwhile demonstrated a carcinogenic effect. ${ }^{36}$ For this reason, the upcoming research will further define the optimal pigment-laser combination for assuring the biocompatibility of this technique.

Shortly after irradiation, there was no increase in ABR thresholds. This finding was not dependent on the intensity of light applied confirming the decreased chance of toxicity with our used energy levels. Further experiments are however needed to clearly define the long-term effects for the hearing system including the testing of each ear independently.

\section{Conclusion}

We were able to induce a collagen remodeling through laser irradiation and histologically prove an increase in collagen in defined areas of the TM. Using a pigment that increased the absorption of photons we reduced the amount of needed light energy and left the structure of the rest of the TM unaffected. This method would allow keeping the natural undamaged structure of the TM while acting in a therapeutic way just onto the pathological changed areas of the TM.

The best increase in TM thickness after laser irradiation of the stained TM could be observed and quantified within the first 4 weeks with no further statistically significant changes afterward in our dataset. Additionally, the intensity of irradiation seemed to be important, just up to $25 \mathrm{~mW}$ for $30 \mathrm{~s}(0.75 \mathrm{~J})$, with no need of further increase in the applied energy. This is due to no further significant TM thickness changes, however, the risk of toxicity.

To our knowledge, this is the first study reporting histological analyses of laser-induced changes within the TM. Additionally, the electrophysiological measurements that demonstrated no irradiation-dependent threshold shift sustain the noninvasive 
therapeutic character of this method. Further studies are on the way to analyze the local temperature increase that induces the collagen remodeling, the long-term effects of this therapy, as well as the exact stiffness of the eardrum after irradiation. Based on these studies, the optimal pigment/laser-parameters to be used could be optimized. Additionally, the study of these effects on an animal model of deficient TM would further complete the information for the clinicians.

\section{Disclosures}

The authors have no relevant financial interests in this article and no potential conflicts of interests to disclose.

\section{Acknowledgments}

This research has been funded by the European Research Council under the European Union's Seventh Framework Program (FP/2007-2013)/ERC Grant, Laser Hearing Aids: 311469. We are grateful to Isaac Ayala Design for the art work and to Dr. Dietmar Hecker for help with setting up the electrophysiology system.

\section{References}

1. M. Schünke et al., PROMETHEUS LernAtlas der Anatomie; Kopf, Hals und Neuroanatomie, 2nd ed., Georg Thieme, Stuttgart (2009).

2. H. Wullstein, "Theory and practice of tympanoplasty," Laryngoscope 66(8), 1076-1093 (1956).

3. M. M. de Araújo et al., "Spontaneous healing of the tympanic membrane after traumatic perforation in rats," Braz. J. Otorhinolaryngol. 80(4), 330-338 (2014).

4. K. Stenfeldt, C. Johansson, and S. Hellström, "The collagen structure of the tympanic membrane: collagen types I, II, and III in the healthy tympanic membrane, during healing of a perforation, and during infection," Arch. Otolaryngol. Head Neck Surg. 132(3), 293-298 (2006).

5. F. Geneser, Color Atlas of Histology, Munksgaard, Kopenhagen (1989).

6. U. Welsch and T. Deller, Lehrbuch Histologie, 3rd ed., Urban \& Fischer, München (2010).

7. D. Lim, "Tympanic membrane: electron microscopic observation part I: pars tensa," Acta otolaryngol. 66(1-6), 181-198 (1968).

8. D. Lim, "Human tympanic membrane: an ultrastructural observation," Acta otolaryngol. 70(3), 176-186 (1970).

9. P. Govaerts, W. Jacob, and J. Marquet, "Histological study of the thin replacement membrane of human tympanic membrane perforations," Acta otolaryngol. 105(3-4), 297-302 (1988).

10. M. Sunita, "Atropic tympanic membrane and hearing assessment," Ind. J. Otolaryngol. Head Neck Surg. 64(1), 22-24 (2012).

11. R. Probst, G. Grevers, and H. Iro, Hals-Nasen-Ohren-Heilkunde, 3rd ed., Thieme, Stuttgart (2008).

12. M. Ryan and D. Kaylie, " $\mathrm{CO}_{2}$ laser myringoplasty: a minimally invasive technique for treating tympanic membrane atelectasis," Otol. Neurotol. 34(9), 1694-1698 (2013).

13. H. Kurkowa and R. L. Goode, "Treatment of tympanic membrane retraction with the holmium laser," Otolaryngol. Head Neck Surg. 112(4), 512-519 (1995).

14. V. B. Ostrowski and D. I. Bojrab, "Minimally invasive laser contraction myringoplasty for tympanic membrane atelectasis," Otolaryngol. Head Neck Surg. 128(5), 711-718 (2003).

15. J. T. Brawner, J. E. Saunders, and W. E. Berryhill, "Laser myringoplasty for tympanic membrane atelectasis," Otolaryngol. Head Neck Surg. 139(1), 47-50 (2008).
16. G. I. Wenzel et al., "Laser irradiation of the Guinea pig basilar membrane," Lasers Surg. Med. 35(3), 174-180 (2004).

17. M. N. Asiyo-Vogel et al., "Histologic analysis of thermal effects of laser thermokeratoplasty and corneal ablation using Sirius-red polarization microscopy," J. Cataract Refractive Surg. 23(4), 515-526 (1997).

18. K. Hayashi et al., "The biologic response to laser thermal modification in an in vivo sheep model," Clin. Orthop. Relat. Res. 373, 265-276 (2000).

19. K. Hayashi et al., "Effect of nonablative laser energy on the joint capsule: an in vivo rabbit study using a holmium: YAG laser," Lasers Surg. Med. 20(2), 164-171 (1997).

20. D. T. Fung et al., "Therapeutic low energy laser improves the mechanical strength of repairing medial collateral ligament," Lasers Surg. Med. 31(2), 91-96 (2002).

21. G. Kesler et al., "Differences in histochemical characteristics of gingival collagen after ER: YAG laser periodontal plastic surgery," J. Clin. Laser Med. Surg. 18(4), 203-207 (2000).

22. J. Tang et al., "Fluorescence spectroscopic imaging to detect changes in collagen and elastin following laser tissue welding," J. Clin. Laser Med. Surg. 18(1), 3-8 (2000).

23. M. A. Trelles et al., "Pulsed and scanned carbon dioxide laser resurfacing 2 years after treatment: comparison by means of scanning electron microscopy," Plast. Reconstr. Surg. 111(6), 2069-2078; discussion 2079-2081 (2003).

24. B. R. Moody, J. E. McCarthy, and G. J. Hruza, "Collagen remodeling after 585-nm pulsed dye laser irradiation: an ultrasonographic analysis," Dermatol. Surg. 29(10), 997-1000 (2003).

25. E. L. Tanzi, C. M. Williams, and T. S. Alster, "Treatment of facial rhytides with a nonablative 1, 450-nm diode laser: a controlled clinical and histologic study," Dermatol. Surg. 29(2), 124-128 (2003).

26. G. I. Wenzel et al., "Laser-induced collagen remodeling and deposition within the basilar membrane of the mouse cochlea," J. Biomed. Opt. 12(2), 021007 (2007).

27. G. Vinitha and A. Ramalingam, "Nonlinear studies of acid Fuchsin dye in liquid and solid media," Spectrochim. Acta, Part A 69(4), 1160-1164 (2008).

28. K. Kowalyk and M. J. Myers, "Dental laser apparatus and method for treating tooth decay," U. S. Patent No. US5456603A (1995).

29. A. Zuccotti et al., "Lack of brain-derived neurotrophic factor hampers inner hair cell synapse physiology, but protects against noise-induced hearing loss," J. Neurosci. 32(25), 8545-8553 (2012).

30. L. Junqueira, W. Cossermelli, and R. Brentani, "Differential staining of collagens type I, II and III by Sirius Red and polarization microscopy," Arch. Histol. Jpn. 41(3), 267-274 (1978).

31. F. Sweat, H. Puchtler, and S. I. Rosenthal, "Sirius red F3BA as a stain for connective tissue," Arch. Pathol. 78, 69-72 (1964).

32. L. C. U. Junqueira, G. Bignolas, and R. Brentani, "Picrosirius staining plus polarization microscopy, a specific method for collagen detection in tissue sections," Histochem. J. 11(4), 447-455 (1979).

33. B. D. C. Vidal, M. L. S. Mello, and É. R. Pimentel, "Polarization microscopy and microspectrophotometry of Sirius Red, Picrosirius and Chlorantine Fast Red aggregates and of their complexes with collagen," Histochem. J. 14(6), 857-878 (1982).

34. G. E. Piérard, "Sirius red polarization method is useful to visualize the organization of connective tissues but not the molecular composition of their fibrous polymers," Matrix 9(1), 68-71 (1989).

35. Y. Hahn et al., "Histologic changes after $\mathrm{CO}_{2}$ laser myringoplasty in Gerbils," Otolaryngol. Head Neck Surg. 143(2 Suppl.), P236 (2010).

36. G. F. Rubino et al., "The carcinogenic effect of aromatic amines: an epidemiological study on the role of o-toluidine and 4, 4'-methylene bis (2-methylaniline) in inducing bladder cancer in man," Environ. Res. 27(2), 241-254 (1982).

Biographies of the authors are not available. 